\title{
Planning Long Dynamically-Feasible Maneuvers for Autonomous Vehicles
}

\author{
Maxim Likhachev \\ School of Computer Science \\ University of Pennsylvania \\ Philadelphia, PA \\ maximl@seas.upenn.edu
}

\author{
Dave Ferguson \\ Intel Research Pittsburgh \\ 4720 Forbes Ave \\ Pittsburgh, PA \\ dave.ferguson@intel.com
}

\begin{abstract}
In this paper, we present an algorithm for generating complex dynamically-feasible maneuvers for autonomous vehicles traveling at high speeds over large distances. Our approach is based on performing anytime incremental search on a multiresolution, dynamically-feasible lattice state space. The resulting planner provides real-time performance and guarantees on and control of the suboptimality of its solution. We provide theoretical properties and experimental results from an implementation on an autonomous passenger vehicle that competed in, and won, the Urban Challenge competition.
\end{abstract}

\section{INTRODUCTION}

Autonomous vehicles navigating through cluttered, unstructured environments or parking in parking lots often need to perform complex maneuvers and reason over large distances. Furthermore, this reasoning usually needs to be performed very quickly so that the resulting maneuvers can be executed in a timely manner, particularly if the environment is inhabited, dynamic, or dangerous. In particular, our current focus is planning for autonomous urban driving including both off-road scenarios and large unstructured parking lots such as the ones in front of malls and large stores (on the order of $200 \times 200$ meters). Maneuvering at human driving speeds ( $\sim 15 \mathrm{mph}$ ) through such areas requires very efficient planning, especially if they contain static obstacles or other moving vehicles.

Roboticists have concentrated on the problem of mobile robot navigation for several decades, providing a large body of research. Early approaches concentrated on local planning, where very short term reasoning is performed to generate the next action for the vehicle. These include potential fieldbased techniques, where obstacles exert repulsive forces on the vehicle while the goal exerts an attractive force [1], and the curvature velocity [2] and dynamic window [3] approaches, where planning is performed in control space to generate dynamically-feasible actions. One major limitation of these purely local approaches was their capacity to get the vehicle stuck in local minima en route to the goal (for instance, cul-desacs). Further, these approaches are unable to perform complex multi-stage maneuvers, such as three-point turns, as these maneuvers are not within the set of local actions considered by the planner.

To reduce the susceptibility to local minima of these approaches, algorithms were developed that incorporated global as well as local information [4, 5, 6, 7]. Typically, these approaches generate a set of candidate simple local actions and evaluate each based on both their local traversability cost and the desirability of their endpoints based on a global value function (e.g. the expected distance to the goal based on known obstacle information). Although these approaches perform better with respect to local minima, their simple local planning can still cause the vehicle to get stuck or take highly suboptimal paths. Subsequent approaches have focused on improving this local planning by using more sophisticated local action sets that better follow the global value function [8, 9], and by generating sequences of actions to perform more complex local maneuvers $[10,11,12]$. The most complex of these approaches are able to perform very precise local maneuvering but are limited by the mismatch between their powerful local planning and their approximate global planning, resulting once more in a susceptibility to local minima.

Recognizing this mismatch, other researchers have concentrated on improving the quality of global planning, so that a global path can be easily tracked by the vehicle $[13,14,15$, $16,17]$. However, the computational expense of generating complex global plans over large distances has remained very challenging, and these approaches are restricted to either small distances, fairly simple environments, or highly suboptimal solutions.

In this paper, we present an efficient, global planning approach that attempts to overcome these challenges. First, we employ a multi-resolution lattice search space to reduce the complexity of the global search while still providing extremely high-quality solutions. Second, we use an efficient anytime, incremental search to quickly generate bounded suboptimal solutions, then improve these solutions while deliberation time allows and repair them when new information is received. The resulting approach is able to plan complex, dynamicallyfeasible maneuvers over hundreds of meters and improve and repair them in real-time for vehicles traveling at high $(\backsim 15$ mph) speeds.

We first describe the key ideas and components of our approach, then provide key theoretical properties and results from both simulation and the Urban Challenge competition. 


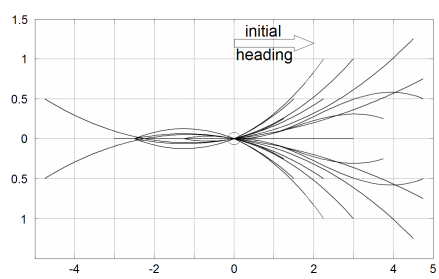

(a) high-resolution

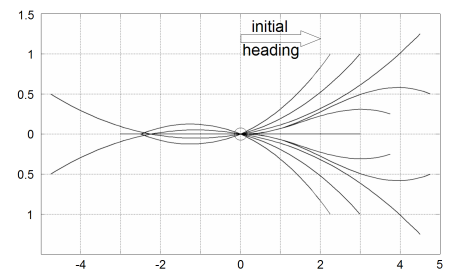

(b) low-resolution
Fig. 1. High- and low-resolution action spaces.

\section{Multi-Resolution Lattice State-space}

A state lattice [18] is a discretization of the configuration space into a set of states, representing configurations, and connections between these states, where every connection represents a feasible path. As such, lattices provide a method for motion planning problems to be formulated as graph searches. However, in contrast to many graph-based representations (such as 4-connected or 8-connected grids), the feasibility requirement of lattice connections guarantees that any solutions found using a lattice will also be feasible. This makes them very well suited to planning for non-holonomic and highly-constrained robotic systems, such as passenger vehicles.

State-space. The two key considerations in constructing a lattice are the discretization (or sampling) strategy used for representing the states in the lattice, and the action space (or control set) used for the inter-state connections. For our application we employ a four dimensional $(x, y, \theta, v)$ state representation, where $(x, y)$ represent the position of the center of the vehicle in the world, $\theta$ represents the orientation of the vehicle, and $v$ represents its translational velocity. The $(x, y, \theta)$ coordinates are important for computing the validity of the poses of the vehicle in the world and making sure that no path in the lattice requires an instantaneous change in the orientation of the vehicle. For the velocity $v$ we use two possible values: maximum forwards velocity and maximum reverse velocity. We take velocity into account because the time involved in switching between forward and backward directions is substantial so reasoning about this cost is important for generating fast, smooth paths ${ }^{1}$.

Action Space. The action space for each state in the lattice is intended to be dense enough that every possible feasible path through the lattice can be constructed by combining sequences of these actions. However, because this action space represents the branching factor of the subsequent graph search, in practice it must be carefully constructed to provide flexibility in path selection while maintaining computational tractability.

The offline construction of our action space is based on work by Pivtoraiko and Kelly [18] that attempts to create nearminimal spanning action spaces. Given a state $s$, we compute the action space by first calculating a subset of states within a distance $d$ of $s$ that are reachable via some feasible action.

\footnotetext{
${ }^{1}$ We do not reason about curvature (the orientation of wheels) because we found this to be less critical for the speeds we are interested in traveling at, as discussed in the results section.
}

To generate the feasible actions we use a trajectory generation algorithm originally developed by Howard and Kelly [9]. This algorithm employs an accurate vehicle model to produce feasible, directly-executable actions and an optimization technique to minimize the endpoint error of these actions with respect to a desired endpoint state. We use this approach to 'snap' the actions to the lattice so that the endpoint of each action lands on a lattice state. Next, we look at this set of actions and calculate whether any single action can be approximately recomposed out of a combination of other, shorter actions. If so, these longer actions are discarded from our set. This provides us with a compact set of actions that approximate the full reachable space. However, in contrast to the approach in [18], we maintain multiple straight segments of varying lengths to improve the speed of the subsequent search, as we will discuss in Section III-A. Figure 1(a) illustrates the action space for a single state (oriented to the right) in our lattice.

Multi-resolution Lattice. Even with a compact action space, planning long complex maneuvers over lattices can be expensive in terms of both computation and memory. An important observation, however, is that usually, there exists a wide spectrum of smooth, dynamically-feasible paths between the vehicle and goal configurations and it is waste of time and memory to explore all of them. On the other hand, all of these paths start and end at the exact same configurations, and the challenge is in finding a path that satisfies the current vehicle configuration and the specific goal configuration precisely.

This motivated us to take a novel, multi-resolution approach, where we use a high-resolution action space in the vicinity of the robot and the goal, and a low-resolution action space elsewhere. We call the resulting combination a multi-resolution lattice. With this approach, we can harness most of the benefit of the high-resolution representation without paying anything near the full computational cost. The trick is making sure that the high-resolution and low-resolution lattices connect together smoothly.

Our multi-resolution approach maintains the same dimensionality $(x, y, \theta, v)$ for both resolutions, but the action space for the low-resolution lattice is a strict subset of the action space for the high-resolution lattice. Figure 1(a) shows the action space used in the high-resolution lattice and Figure 1(b) shows the action space used in the low-resolution lattice ${ }^{2}$. Using this method ensures that the low-resolution lattice is utilized fully and that paths in the multi-resolution lattice are guaranteed to be feasible, which is a strong advantage over existing combined local and global approaches for navigation.

Theorem 1: Every path in a lattice that uses only a lowresolution action space is also a valid path in our multiresolution lattice. Further, every path in the multi-resolution lattice is a valid path in a lattice that uses only the highresolution action space.

Proof. The proof of the first claim follows trivially from the fact that any action in the low-resolution lattice is a valid

\footnotetext{
${ }^{2}$ In practice, choosing the appropriate set can be achieved with a basic check: if the $(x, y)$ location of a state is not within some distance $d$ of the vehicle or goal, its action set is the low-resolution set.
} 
action in both the low-resolution and high-resolution lattices, and therefore is a valid action in the multi-resolution lattice. A similar argument applies for the second claim.

Enforcing the low-resolution action space to be a subset of the high-resolution action space decreases the branching factor of the graph constructed by the search, which is certainly important, but it does not necessarily decrease the size of the graph. However, it is also possible to decrease the size of the graph as follows. Suppose $A_{h}$ is an action space used in the high-resolution space, and $A_{l}$ is an action space used in the low-resolution space. Thus, $A_{l} \subset A_{h}$. Then, we can construct $A_{l}$ by picking only the actions from $A_{h}$ that end at states with a coarser discretization than the end states of actions in $A_{h}$. For example, we can choose for $A_{l}$ only those actions whose end states have $\theta$ equal to one of 16 possible angles, while actions in $A_{h}$ can connect states with 32 possible values of $\theta$. (This is precisely what we used in our system.) Mathematically, the construction of the action space $A_{l}$ can be expressed as follows in terms of a high-resolution discretization $Q_{h}$ and a lower-resolution discretization $Q_{l}$ of variables $x, y, \theta, v$ : an action $a$ connecting states $s_{1}=\left(x_{1}, y_{1}, \theta_{1}, v_{1}\right)$ and $s_{2}=$ $\left(x_{2}, y_{2}, \theta_{2}, v_{2}\right)$ belongs to $A_{l}$ if and only if $a \in A_{h}$ and $\left(x_{2}, y_{2}, \theta_{2}, v_{2}\right) \in Q_{l}$.

Restricting $Q_{l}$ to a coarser discretization for $(x, y)$ or $\theta$ corresponds to using a discretization that adapts based on the vehicle and goal configurations. This technique can also be used to explicitly constrain the behavior of the vehicle in the different areas. For instance, restricting $Q_{l}$ to contain only positive $v$-values prevents the vehicle from moving backward when far from the initial and goal configurations. This general approach allows for an arbitrarily-reduced state and action space in the low-resolution portion of the lattice, and can also be trivially extended to more than two levels of resolution if desired.

\section{Anytime, InCREMEntal SEARCH}

Given a search space (in our case, in the form of a multiresolution lattice) and a cost function associated with each action, we need an efficient method for searching through this space for a solution path. $A^{*}$ search is perhaps one of the most popular methods for doing this [19]. It utilises a heuristic to focus the search towards the most promising areas of the search space. While highly efficient, $A^{*}$ aims to find an optimal path which may not be feasible given time constraints and the size of environments autonomous vehicles need to operate in. To cope with very limited deliberation time, anytime variants of $\mathrm{A}^{*}$ search have been developed [20, 15]. These algorithms generate an initial, possibly highlysuboptimal solution very quickly and then concentrate on improving this solution while deliberation time allows. Furthermore, these anytime algorithms are able to provide bounds on the suboptimality of the solution at any point of time during the search.

$\mathrm{A}^{*}$ and its anytime variants work best when the search space, and thus environment, is mostly known a priori. In
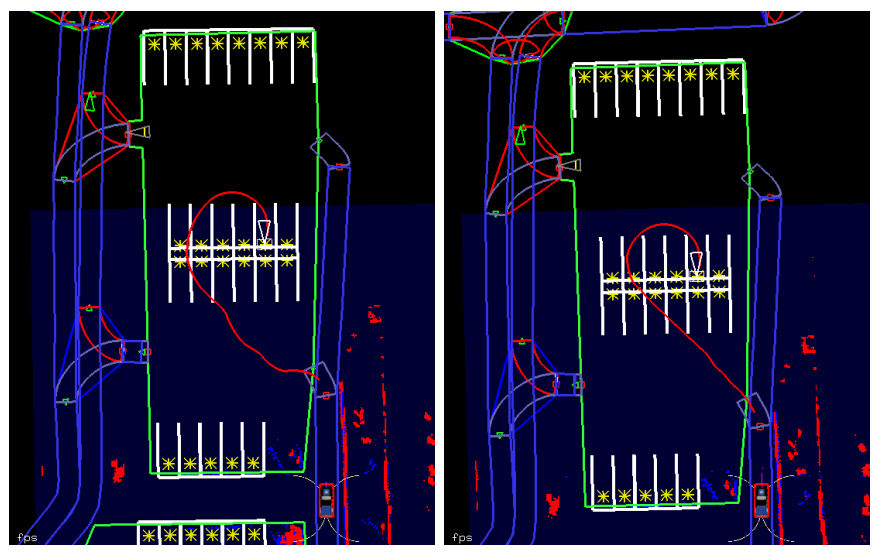

Fig. 2. Pre-planning a path into a parking spot and improving this path in an anytime fashion.

robotic path planning this is rarely the case, and the robot typically receives updated environmental information through onboard and/or offboard sensors during execution. To cope with imperfect initial information and dynamic environments, efficient incremental variants of $\mathrm{A}^{*}$ search have been developed that update previous solutions based on new information (e.g. from sensors) [21, 22, 23]. These algorithms repair existing solutions for a fraction of the computation required to generate such solutions from scratch.

When faced with limited deliberation time and imperfectlyknown or dynamic environments, it is extremely useful to have a search algorithm that is both anytime and incremental. The Anytime Dynamic A* algorithm developed by Likhachev et al. is a version of $A^{*}$ search that combines these two properties into a single approach and has been shown to be very effective for a range of robotic planning tasks [16]. We employ this algorithm for planning and re-planning paths in our multiresolution lattice.

\section{A. Anytime Dynamic A*}

Anytime Dynamic $A^{*}\left(\mathrm{AD}^{*}\right)$ exploits a property of $\mathrm{A}^{*}$ that can result in much faster generation of solutions, namely that if consistent heuristics are used and multiplied by an inflation factor $\epsilon>1$, then $\mathrm{A}^{*}$ can often generate a solution much faster than if no inflation factor is used [24], and the cost of the solution generated by $\mathrm{A}^{*}$ will be at most $\epsilon$ times the cost of an optimal solution [25]. AD* operates by performing a series of these inflated $A^{*}$ searches with decreasing inflation factors, where each search reuses information from previous searches. By doing so, it is able to provide suboptimality bounds on all solutions generated and allows for control of these bounds, since the user can decide how much the inflation factor is decreased between searches. To cope with updated information, $\mathrm{AD}^{*}$ also borrows ideas from the $\mathrm{D}^{*}$ and $\mathrm{D}^{*}$ Lite algorithms [21, 22] and only propagates updated information through the affected and relevant (given the current search) portions of the search space.

To enable efficient anytime planning and replanning as the vehicle moves, we use $\mathrm{AD}^{*}$ to search backwards from the goal 
configuration towards the current configuration of the vehicle. The heuristic used thus needs to estimate the cost of a shortest path from the vehicle configuration (rather than goal) to each state in question.

The effectiveness of Anytime Dynamic A* is highly dependent on its use of an informed heuristic to focus its search. An accurate heuristic can reduce the time and memory required to generate a solution by orders of magnitude, while a poor heuristic can diminish the benefits of the algorithm. It is thus important to devote careful consideration to the heuristic used for a given search space. Further, because we are inflating heuristic values, it is useful to have long actions that can skip over several nodes and reduce the number of states in the search. It is for this reason we add several straight line actions of varying length in both the forwards and backwards directions to our action set (see Section II).

\section{B. Informative Heuristics}

The purpose of a heuristic is to improve the efficiency of the search by guiding it in promising directions. A common approach for constructing a heuristic is to use the results from a simplified search problem (e.g. from a lower-dimensional search problem where some of the original constraints have been relaxed). In selecting appropriate heuristics, it is important to analyze the original search problem and determine the key factors contributing to its complexity. In robotic path planning these are typically the complexity inherited from the constraints of the mechanism and the complexity inherited from the nature of the environment.

To cope with the complexity inherited from the mechanism constraints, a very useful general heuristic is the cost of an optimal solution through the search space assuming a completely empty environment. This can be computed offline and stored as a heuristic lookup table, and several efficiencies can be used to reduce the required memory for this table [17]. This is a very well informed heuristic for operating in sparse environments and is guaranteed to be an optimistic (or admissible) approximation of the actual path cost.

To cope with the complexity inherited from the nature of the environment, it is not practical to pre-compute heuristic values for all possible environment configurations, as there are an effectively infinite number of possibilities for any reasonablysized environment. However, in this case it is beneficial to solve online a simplified search problem given the actual environment and use the result of this search as a heuristic to guide the original, complex search. In particular, we solve a 2D $((x, y))$ version of the problem by running a single Dijkstra's search starting at the cell that corresponds to the center of the current vehicle position. The search computes the costs of shortest paths to all other cells in the environment ${ }^{3}$.

$\mathrm{AD}^{*}$ requires the heuristics to be admissible and consistent. This holds if $h\left(s_{\text {start }}\right)=0$ and for every pair of states $s, s^{\prime}$ such that $s^{\prime}$ is an end state of a single action executed at state

\footnotetext{
${ }^{3}$ However, even though it is very fast, we still restrict this search to only compute the states that are no more than twice as far (in terms of path cost) from the vehicle cell as the goal cell.
}

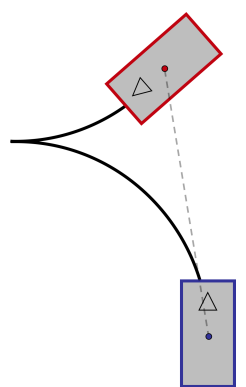

(a)

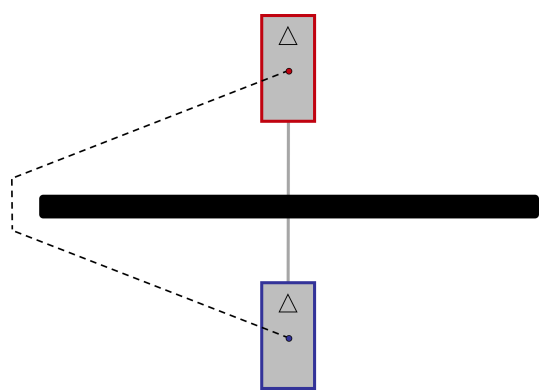

(b)
Fig. 3. Mechanism-constrained (solid) and environment-constrained (dashed) heuristic paths. In each case, the initial and desired vehicle poses are shown as blue and red rectangles, respectively (with the interior triangles specifying the headings). (a) The mechanism-constrained heuristic is perfectly informed when no obstacles are present in the environment. (b) The environmentconstrained 2D heuristic can provide significant benefit when obstacles exist. Here, an obstacle (shown in black) resides over the direct path to the desired pose.

$s, h(s)+c\left(s, s^{\prime}\right) \geq h\left(s^{\prime}\right)$, where $h(s)$ is a heuristic of state $s$, $s_{\text {start }}$ is a state that corresponds to the vehicle configuration and $c\left(s, s^{\prime}\right)$ is the cost of the action that connects $s$ to $s^{\prime}$. The cost $c\left(s, s^{\prime}\right)$ of the action is typically computed as the length of the action times the average of the costs of the cells covered by the vehicle when moving from state $s$ to state $s^{\prime}$. The heuristic based on the 2D search, however, may overestimate these costs since it estimates the cost of moving the center of the vehicle only. To resolve this, the cost of each cell in the $2 \mathrm{D}$ grid used for computing the $2 \mathrm{D}$ heuristic is set to the average of cells covered by the largest circle than can be inscribed into the vehicle perimeter. The cost of each transition $c\left(s, s^{\prime}\right)$ is then computed as the length of the transition times the maximum of two quantities: (a) the average value of the costs of the cells covered by the vehicle when moving from state $s$ to state $s^{\prime}$ (as before), and (b) the maximum of the 2D grid cell costs, used to compute heuristics, traversed through by the center of the vehicle when moving from $s$ to $s^{\prime}$. Intuitively, this cost function penalizes slightly more when vehicle traverses highcost areas (e.g., obstacles) residing right under the center of the vehicle. In addition, the heuristics are scaled down by a factor of 1.08 to compensate for the suboptimality of optimal paths in 8-connected grids. It can be then shown that our 2D heuristic function is admissible and consistent with respect to this cost function.

Each of these heuristic generation approaches, mechanismrelative and environment-relative, have strong and complementary benefits (see Figure 3). Rather than selecting one, it is possible to combine the two. We do this by constructing a new heuristic that, for each state $s$, returns the value $h(s)=$ $\max \left(h_{f s h}(s), h_{2 D}(s)\right)$, where $h_{f s h}(s)$ is the heuristic value of state $s$ according to the mechanism-constrained heuristic (freespace heuristic), and $h_{2 D}(s)$ is the value according to the environment-constrained heuristic (2D heuristic). As shown in the experimental results, this combined heuristic function can be an order of magnitude more effective than either of the component heuristic functions. Since both $h_{f s h}(s)$ and 
$h_{2 D}(s)$ are admissible and consistent, the combined heuristic is also admissible and consistent [26]. This property implies the bounds on the suboptimality of the paths returned by $\mathrm{AD}^{*}[16]$ :

Theorem 2: The cost of a path returned by Anytime Dynamic $\mathrm{A}^{*}$ is no more than $\epsilon$ times the cost of a least-cost path from the vehicle configuration to the goal configuration using actions in the multi-resolution lattice, where $\epsilon$ is the current value by which Anytime Dynamic A* inflates heuristics.

\section{OPTIMIZATIONS}

Typically, one of the most computationally expensive parts of planning for vehicles is computing the cost of actions, as this involves convolving the geometric footprint of the vehicle for a given action with a map from perception. In our application, we used a $0.25 \mathrm{~m}$ resolution $2 \mathrm{D}$ perception map and the $(x, y)$ dimensions of our vehicle were $5.5 \mathrm{~m} \times 2.25 \mathrm{~m}$. Thus, even a short $1 m$ action requires collision checking roughly 300 cells. Further, the specific cells need to be calculated based on the action and the initial pose of the vehicle.

To reduce the processing required for this convolution, we performed two optimization steps. First, for each action $a$ we pre-computed the cells covered by the vehicle when executing this action. During online planning, these cells are quickly extracted and translated to the appropriate position when needed. Second, we generated two configuration space maps to be used by the planner to avoid performing convolutions. The first of these maps expanded all obstacles in the perception map by the inner radius of the robot; this map corresponded to an optimistic approximation of the actual configuration space. Given a specific action $a$, if any of the cells through which the center of the robot executing action $a$ passes are obstacles in this inner map, then $a$ is guaranteed to collide with an obstacle. The second map expanded all obstacles in the perception map by the outer radius of the robot and therefore corresponded to a pessimistic approximation of the configuration space. If all of the cells through which the center of the vehicle passes when executing action $a$ are obstacle-free in this map, then $a$ is guaranteed to be collision-free. Only those actions that do not produce a conclusive result from these simple tests need to be convolved with the perception map. Typically, this is a severely reduced percentage, thus saving considerable computation. To create these auxiliary maps efficiently, we performed a single distance transform on the perception map and then thresholded the distances using the corresponding radii of the robot for each map.

\section{EXPERIMENTAL RESUlts}

We have implemented our approach on an autonomous passenger vehicle (lower-left image in Figure 5) where it has been used to drive over 3000 kilometers in urban environments, including competing in the DARPA Urban Challenge. The multi-resolution lattice planner was used for planning through parking lots and into parking spots, as well as for geometric road following in off-road areas, and in error recovery scenarios. During these scenarios, the vehicle traveled speeds of

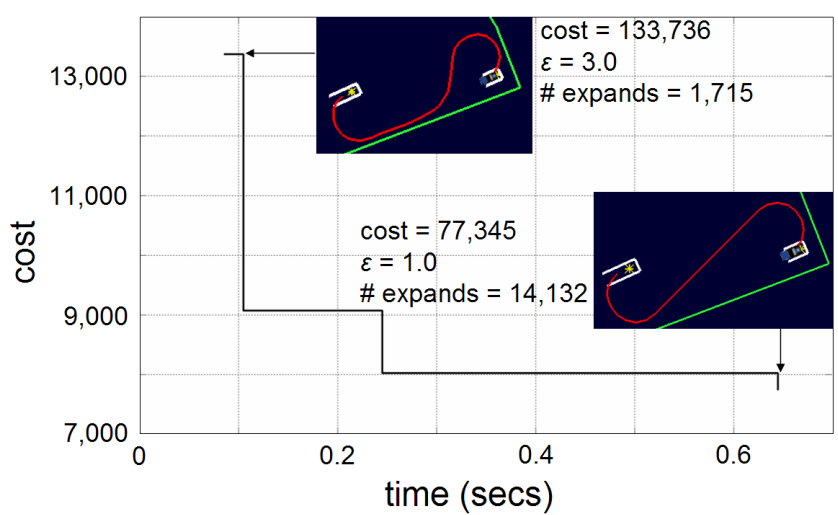

(a) anytime behavior
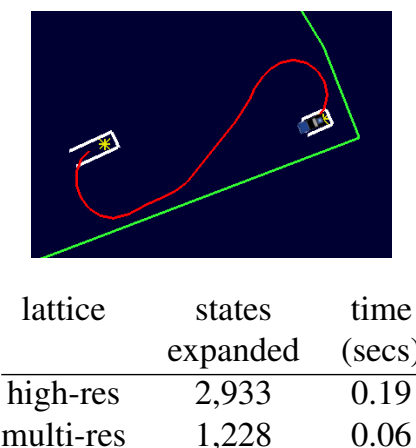

(b) effect of multi-res lattice

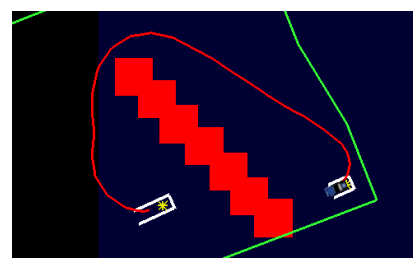

heuristic states time

\begin{tabular}{ccc} 
& expanded & (secs) \\
\hline$h$ & 2,019 & 0.06 \\
$h_{2 D}$ & 26,108 & 1.30 \\
$h_{f s h}$ & 124,794 & 3.49
\end{tabular}

(c) effect of heuristic
Fig. 4. An example highlighting our approach's anytime behavior and the benefits of the multi-resolution lattice and the combined heuristic function.

up to 15 miles per hour while performing complex maneuvers and avoiding static and dynamic obstacles.

In all cases, the multi-resolution lattice planner searches backwards out from the goal pose (or set of goal poses) and generates a path consisting of a sequence of feasible highfidelity maneuvers that are collision-free with respect to the static obstacles observed in the environment. This path is also biased away using cost function from undesirable areas such as curbs and locations in the vicinity of dynamic obstacles.

When new information concerning the environment is received (for instance, a new static or dynamic obstacle is observed), the planner is able to incrementally repair its existing solution to account for the new information. This repair process is expedited by performing the search in a backwards direction, as in such a scenario updated information in the vicinity of the vehicle affects a smaller portion of the search space and so less repair is required. The lattice plan is typically updated once per second, however in trivial or very difficult scenarios this time may vary.

As mentioned earlier, the lattice used in this application does not explicitly represent curvature. Theoretically, this means that the paths produced over this lattice are guaranteed feasible only if we allow the vehicle to stop at each lattice state and re-orient its steering wheel. However, in practice we reduce (by a small fraction) the maximum curvature used 

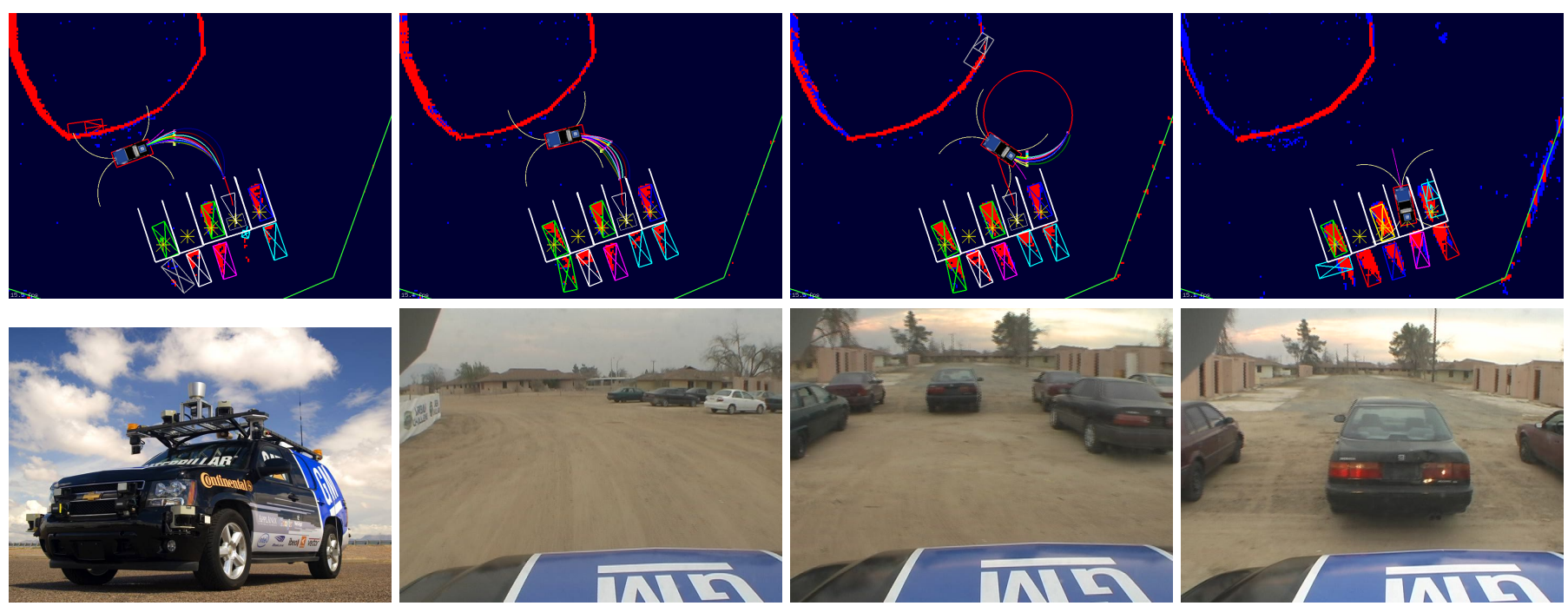

Fig. 5. Replanning when new information is received

in generating connections between states and we reduce the maximum speed at which we execute higher-curvature sections of lattice paths (from $5 \mathrm{~m} / \mathrm{s}$ down to $2 \mathrm{~m} / \mathrm{s}$ ) so that this curvature discontinuity is not a critical issue. We also use a lookahead during execution to slow down and stop when switching velocity directions ${ }^{4}$. As a result, we don't need to stop during execution unless the path contains velocity sign changes.

The lattice path is tracked using a local planner that employs the same trajectory generation algorithm used to provide the action space for the lattice. Although a simple, singletrajectory tracker would suffice given the feasibility of the lattice plan, multiple trajectories are produced to account for dynamic obstacles and new observations that could require immediate reaction (the local planner runs at $10 \mathrm{~Hz}$ ).

To ensure that a high-quality path is available for the vehicle as soon as it enters a parking lot, the lattice planner begins planning for the desired goal pose while the vehicle is still approaching the lot. By planning a path from the entry point of the parking lot in advance, the vehicle can seamlessly transition into the lot without needing to stop, even for very large and complex lots. Further, the anytime property of the search enables the solution to be improved during the pre-planning stage and, depending on how much time is available for pre-planning, the resulting path for the vehicle can converge to a (provably) optimal solution.

As well as providing smooth navigation amongst partiallyknown static objects, the efficiency of the multi-resolution lattice planner makes it possible to intelligently interact with several dynamic obstacles in the environment. In our application, we were able to not only avoid such obstacles but through updating regions of high cost as the obstacles moved, we could stay well clear of them unless necessary and also exhibit intelligent yielding behavior in unstructured areas (e.g. keeping to the right when approaching oncoming vehicles).

\footnotetext{
${ }^{4} \mathrm{~A}$ maximum lookahead of $2 m$ is required given our vehicle's maximum deceleration and the top speed used for following lattice paths, but we use a slightly higher lookahead for smooth deceleration.
}

The multi-resolution lattice planner was also used for performing complex maneuvers in error recovery scenarios during on-road driving, such as when a lane or intersection is partially blocked with vehicles or obstacles, or a road is fully blocked and a u-turn is required. It was also used when there was some uncertainty as to where the road was; in these scenarios it uses the geometric perceptual information to bias the vehicle towards the center of the road (when there are perceivable curbs or berms).

We have included here a number of examples from the Urban Challenge and our testing to illustrate key characteristics of the approach.

a) Pre-planning: Figure 2 illustrates the pre-planning used by the lattice planner, as well as its anytime performance. The left image shows our vehicle approaching a parking lot (parking lot boundary shown in green, road lanes shown in blue), with its intended parking spot indicated by the white triangle. While the vehicle is still outside the parking lot it begins planning a path from one of the parking lot entries to the desired spot (path shown in red). Although the initial path shown in this left image is feasible, it is not ideal as it involves more turning than necessary. The right image shows how this path is improved over time as the vehicle approaches. This path is optimal with respect to our cost function and is generated well before the vehicle enters the parking lot.

b) Anytime Planning: Figure 4 is intended to provide insights into the benefits provided by each of the main components of our approach. Figure 4(a) illustrates the anytime behavior of the approach when planning between two parking spots. We have included a plot of the cost of the solution produced by Anytime $\mathrm{D}^{*}$ as a function of computation time. Here, the initial suboptimality bound $\epsilon$ was set to 3 . The upper image shows the first path Anytime $D^{*}$ finds. This path was found in less than 100 msecs (and after 1,715 state expansions). The cost of the path was 133,736. Given additional deliberation time, Anytime $\mathrm{D}^{*}$ improves upon this solution, and after 650 msecs, the search converges to an optimal solution. This solution is significantly shorter than the 
initial path (as seen in the bottom image) and has a cost of 77,345 .

c) Multi-resolution Planning: Figure 4(b) shows the benefits of using our multi-resolution lattice approach on the same simple example. The top row in the table represents a uniformly high-resolution lattice, while the bottom row represents our multi-resolution lattice (in both cases, $\epsilon=2$ ). Planning with the multi-resolution lattice is more than three times faster. Note that the improvement in states expanded is less than a factor of three. This is because using a multiresolution lattice decreases not only the number of states expanded but also the time spent expanding each state, since the number of possible actions from each state is decreased.

d) Combining Mechanism-relative and Environmentrelative Heuristics: Figure 4(c) demonstrates the benefits of using our combined heuristic function on a simple example. The first row in the table represents our combined heuristic function. It combines the 2D environment-constrained heuristic (2nd row) and freespace mechanism-constrained heuristic (3rd row). Using this combination is over 21 times faster than using the 2D heuristic alone and over 58 times faster than using the freespace heuristic alone.

e) Replanning: Figure 5 illustrates the replanning capability of the lattice planner. These images were taken from a parking task performed during the National Qualification Event. The top-left image shows the initial path planned for the vehicle to enter the parking spot indicated by the white triangle. Several of the other spots were occupied by other vehicles (shown as rectangles of varying colors), with detected obstacles shown as red areas. The trajectories generated to follow the path are shown emanating from our vehicle (the selected trajectory is shown in blue). As the vehicle gets closer to its intended spot, it observes more of the vehicle parked in the right-most parking spot (top, second image from left). At this point, it realizes its current path is infeasible and replans a new path that has the vehicle perform a loop and pull in smoothly. This path was favored in terms of time over stopping and backing up to re-position. The three right-most photographs on the bottom row were taken by an onboard camera during the run.

f) Long-range Planning: As with other teams participating in the Urban Challenge, our vehicle underwent extensive testing before and during the competition. During the competition, the planner was able to continuously plan and replan without having the vehicle ever stop to wait for a plan. The scenarios we used for testing before the competition were numerous and included expansive obstacle-laden parking lots as well as narrow, highly-constrained parking lots. An example of the former is shown in Figure 6(a-b). This parking lot is $200 \mathrm{~m}$ by $200 \mathrm{~m}$. Initially, it is unknown and as the robot traverses the lot, it discovers a series of obstacles (shown as white dots in the image on the right). The robot has to replan in real-time to account for these obstacles. The time for replanning in this scenario varied from a few milliseconds for small re-planning adjustments to the path to a few seconds for finding drastically different trajectories, such as the one shown

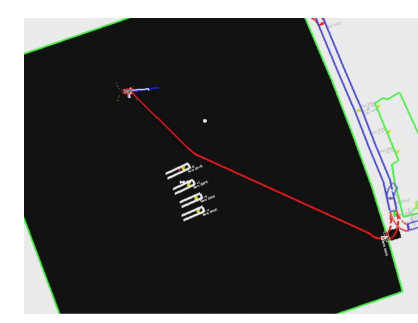

(a) initial planning

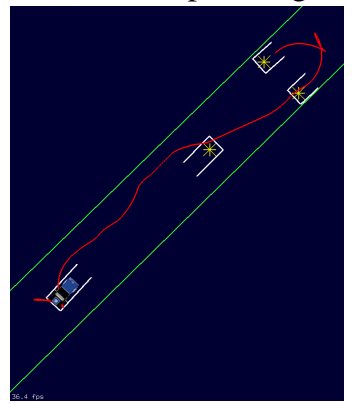

(c) initial planning

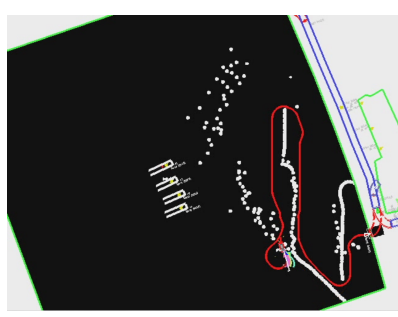

(b) replanning

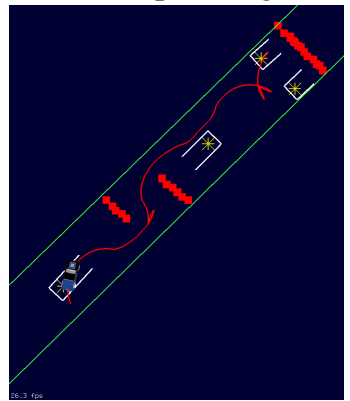

(d) replanning
Fig. 6. Planning and replanning in large $(a, b)$ and highly-constrained $(c, d)$ environments

in Figure 6(b).

g) Complex Maneuvering: An example of a testing scenario involving a highly-constrained parking lot is shown in Figure 6(c-d). The trajectory planned involves the robot making an initial narrow U-turn and then making another one immediately before pulling into the final parking spot. While executing the trajectory, the robot discovers a series of obstacles and has to re-plan as shown in Figure 6(d). The new trajectory now requires the robot to backup a number of times. Moreover, it requires the robot to enter the desired spot in reverse since the discovered obstacles prohibit the robot from pulling in.

h) Coping with Dynamic Obstacles: Figure 7 shows the lattice planner being used to plan amongst several other moving vehicles in simulation. In these images, the current goal is shown as the white triangle and the inferred shortterm trajectories of the other vehicles are included as fading polygons.

i) Coping with Static Obstacles: Figure 8 provides an example testing scenario for our physical vehicle. The left image shows the layout of the parking lot, the static obstacles (initially unknown to the vehicle), and the parking spots to be visited in order ( 1 through 5 ). The vehicle entered the lot through the left entrance between spots 3 and 4 . The other images show snapshots from an onboard camera during the vehicle's traverse through this difficult environment.

\section{CONCLUSIONS}

We have presented a general approach for complex planning involving large, high-dimensional search spaces. Our approach employs a novel multi-resolution action and state space that significantly reduces complexity while providing a seamless interface between the resolutions, as well as guarantees of solution feasibility. The approach also relies 

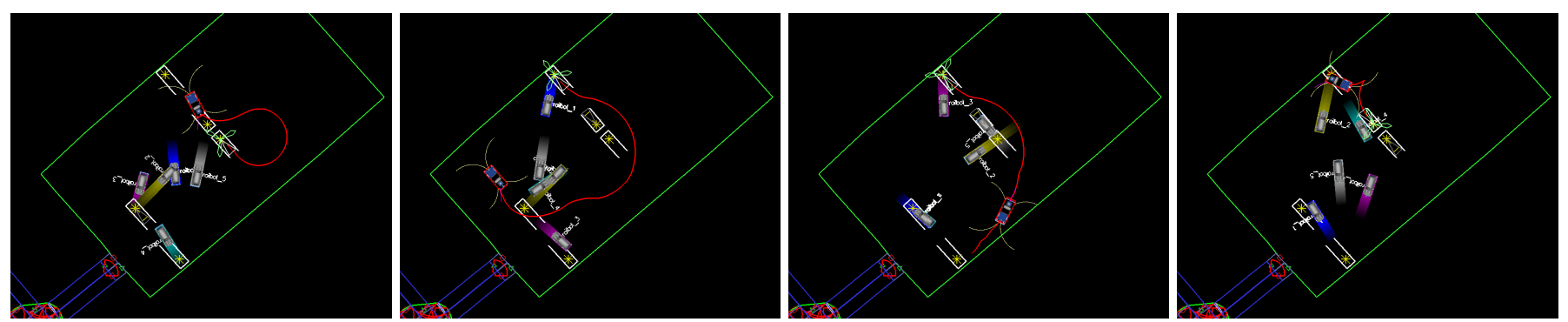

Fig. 7. Planning amongst moving obstacles
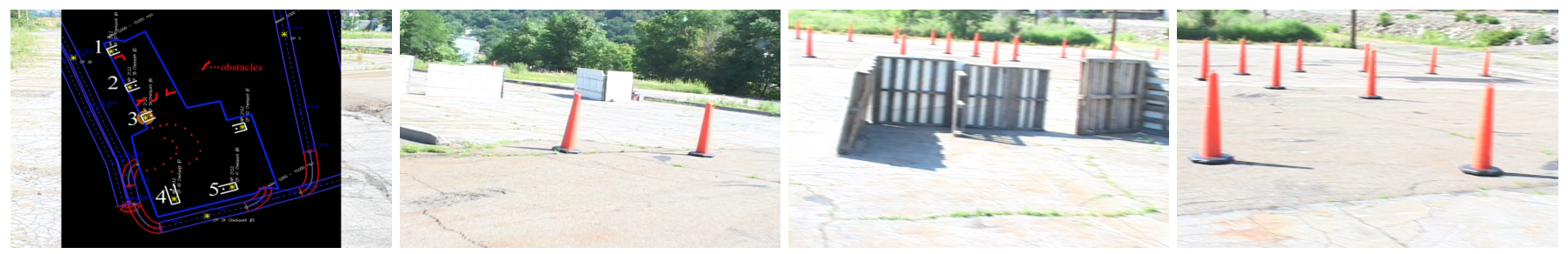

Fig. 8. Planning in complex obstacle environments

on an anytime, incremental search algorithm for generating solutions in partially-known or dynamic environments when deliberation time is limited. This search exploits a lowdimensional environment-dependent heuristic coupled with a full-dimensional freespace heuristic for efficient focusing, a powerful technique applicable to any high-dimensional planning problem. The resulting approach provides global, feasible solutions to challenging navigation tasks, and all the core techniques presented are applicable to a wide range of complex planning problems.

\section{REFERENCES}

[1] O. Khatib, "Real-time obstacle avoidance for manipulators and mobile robots," International Journal of Robotics Research, vol. 5, no. 1, pp. 90-98, 1986.

[2] R. Simmons, "The curvature velocity method for local obstacle avoidance," in Proceedings of the IEEE International Conference on Robotics and Automation (ICRA), 1996.

[3] D. Fox, W. Burgard, and S. Thrun, "The dynamic window approach to collision avoidance." IEEE Robotics and Automation, vol. 4, no. 1, 1997.

[4] S. Thrun et al., "Map learning and high-speed navigation in RHINO," in AI-based Mobile Robots: Case Studies of Successful Robot Systems, D. Kortenkamp, R. Bonasso, and R. Murphy, Eds. MIT Press, 1998.

[5] O. Brock and O. Khatib, "High-speed navigation using the global dynamic window approach," in Proceedings of the IEEE International Conference on Robotics and Automation (ICRA), 1999.

[6] A. Kelly, "An intelligent predictive control approach to the high speed cross country autonomous navigation problem," Ph.D. dissertation, Carnegie Mellon University, 1995.

[7] R. Philippsen and R. Siegwart, "Smooth and efficient obstacle avoidance for a tour guide robot," in Proceedings of the IEEE International Conference on Robotics and Automation (ICRA), 2003.

[8] S. Thrun et al., "Stanley: The robot that won the DARPA Grand Challenge," Journal of Field Robotics, vol. 23, no. 9, pp. 661-692, August 2006.

[9] T. Howard and A. Kelly, "Optimal rough terrain trajectory generation for wheeled mobile robots," International Journal of Robotics Research, vol. 26, no. 2, pp. 141-166, 2007.

[10] C. Stachniss and W. Burgard, "An integrated approach to goal-directed obstacle avoidance under dynamic constraints for dynamic environments," in Proceedings of the IEEE International Conference on Intelligent Robots and Systems (IROS), 2002.

[11] C. Urmson et al., "A robust approach to high-speed navigation for unrehearsed desert terrain," Journal of Field Robotics, vol. 23, no. 8, pp. 467-508, August 2006.
[12] D. Braid, A. Broggi, and G. Schmiedel, "The TerraMax autonomous vehicle," Journal of Field Robotics, vol. 23, no. 9, pp. 693-708, August 2006.

[13] S. LaValle and J. Kuffner, "Rapidly-exploring Random Trees: Progress and prospects," Algorithmic and Computational Robotics: New Directions, pp. 293-308, 2001.

[14] G. Song and N. Amato, "Randomized motion planning for car-like robots with C-PRM," in Proceedings of the IEEE International Conference on Intelligent Robots and Systems (IROS), 2001.

[15] M. Likhachev, G. Gordon, and S. Thrun, "ARA*: Anytime A* with provable bounds on sub-optimality," in Advances in Neural Information Processing Systems. MIT Press, 2003.

[16] M. Likhachev, D. Ferguson, G. Gordon, A. Stentz, and S. Thrun, "Anytime Dynamic A*: An Anytime, Replanning Algorithm," in Proceedings of the International Conference on Automated Planning and Scheduling (ICAPS), 2005.

[17] R. Knepper and A. Kelly, "High performance state lattice planning using heuristic look-up tables," in Proceedings of the IEEE International Conference on Intelligent Robots and Systems (IROS), 2006.

[18] M. Pivtoraiko and A. Kelly, "Generating near minimal spanning control sets for constrained motion planning in discrete state spaces," in Proceedings of the IEEE International Conference on Intelligent Robots and Systems (IROS), 2005.

[19] N. Nilsson, Principles of Artificial Intelligence. Tioga Publishing Company, 1980.

[20] R. Zhou and E. Hansen, "Multiple sequence alignment using A*," in Proceedings of the National Conference on Artificial Intelligence (AAAI), 2002, Student abstract.

[21] A. Stentz, "The Focussed D* Algorithm for Real-Time Replanning," in Proceedings of the International Joint Conference on Artificial Intelligence (IJCAI), 1995.

[22] S. Koenig and M. Likhachev, "Improved fast replanning for robot navigation in unknown terrain," in Proceedings of the IEEE International Conference on Robotics and Automation (ICRA), 2002.

[23] M. Barbehenn and S. Hutchinson, "Efficient search and hierarchical motion planning by dynamically maintaining single-source shortest path trees," IEEE Transactions on Robotics and Automation, vol. 11, no. 2, pp. 198-214, 1995.

[24] J. G. Gaschnig, "Performance measurement and analysis of certain search algorithms," Ph.D. dissertation, Carnegie Mellon University, 1979.

[25] H. W. Davis, A. Bramanti-Gregor, and J. Wang, "The advantages of using depth and breadth components in heuristic search," in Methodologies for Intelligent Systems, 3, Z. W. Ras and L. Saitta, Eds. New York: North-Holland, 1988, pp. 19-28.

[26] J. Pearl, Heuristics: Intelligent Search Strategies for Computer Problem Solving. Addison-Wesley, 1984. 